\title{
A Guide to Read Follow Up MR of Glioblastoma: Tips and
}

\section{Tricks}

\author{
Khalid A and Mubarak F* \\ Department of Radiology, Aga Khan University, Pakistan
}

*Corresponding author: Fatima Mubarak, Department of Radiology, Aga Khan

University, Pakistan, Tel: +92(300)2550951; Email: fatima.mubarak@aku.edu

\section{Mini Review \\ Volume 3 Issue 3}

Received Date: June 11, 2019

Published Date: July 15, 2019

DOI: $10.23880 /$ crij-16000149

\begin{abstract}
Post-surgery prognosis for a brain tumor is influenced as much by histology as by completeness of resection. For the purpose of evaluation of response to treatment, the exact identification of any residual tumor holds central position. A review of the current and past literature addressing the tips and tricks involved in post-operative Magnetic Resonance Imaging (MRI) for the definition of post resection glioblastoma residue is given.
\end{abstract}

Keywords: Postoperative Imaging; Fluid-Attenuated Inversion Recovery; Cerebral Gliomas; Early Post-Operative MR Imaging

Abbreviations: MRI: Magnetic Resonance Imaging; DWI: Diffusion-Weighted Imaging; PWI: PerfusionWeighted Imaging; FLAIR: Fluid-Attenuated Inversion Recovery; ADC: Apparent Diffusion Coefficient; DSC: Dynamic Susceptibility Contrast; rCBV: Relative Cerebral Blood Volume.

\section{Introduction}

Advanced MRI is the primary and most reliable source of Post-operative imaging after brain tumor resection [1,2]. Early post-operative imaging clearly provides clinicians with an edge towards more educational benefits regarding making surgical decisions as well as helps them determine post-treatment response evaluation and incidental tumor progression as compared to late postoperative imaging. Surgery related complications are avoided by putting these advanced imaging techniques to effective use. The most common advanced imaging techniques currently being used include diffusionweighted imaging (DWI) and perfusion-weighted imaging (PWI).
This article provides a review of the various posttreatment-related imaging appearances of brain neoplasms, including a discussion of advanced MR imaging techniques available and treatment response criteria most commonly used in clinical practice.

\section{Review of Evidence}

Contrast-enhanced T1-weighted MRI imaging is the workhorse of brain tumor imaging. Four types of enhancement have been described by Knauth $M$, et al. following brain tumor resections [3]. Meningeal enhancement seen as linear enhancement of the lepto and/or pachy meninges in the immediate post-operative period. The intensity of post-operative meningeal enhancement may increase for up to three days and usually vanishes within one year after surgery but may persist for decades $[4,5]$. Increased enhancement of the ipsilateral choroid plexus in comparison with the contralateral side has been described after opening of the lateral ventricle during surgery [6]. Linear enhancement at the resection margins. Immediate intraparenchymal 


\section{Clinical Radiology \& Imaging Journal}

enhancement having the same appearance and time course as enhances residual tumor. In such cases, a comparison with the preoperative MR examination is essential [6] (Figure 1).

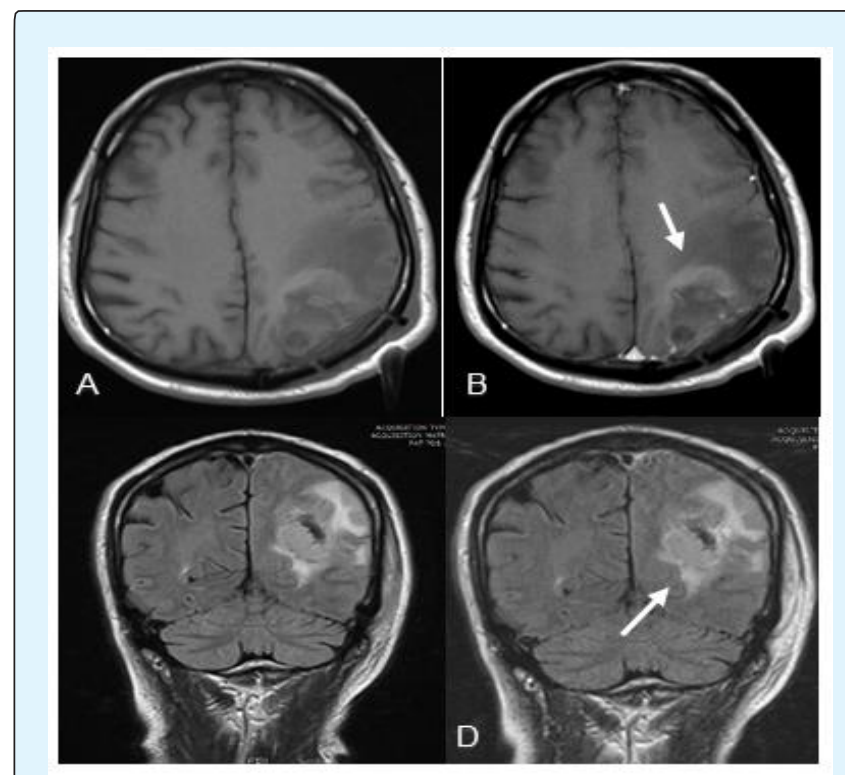

Figure 1: EPMRI (within 48 hours post op) A: Axial T1 - weighted B: Axial T1-post contrast C: Coronal T2 weighted D: Coronal T2 post contrast Status post grade IV glioblastoma resection Abnormal signals along the inferior aspect as well as along the periphery of resection cavity with mild enhancement suggestive of residual disease.

According to Albert FK, et al. Contrast-enhanced T1weighted MRI is extremely valuable for assessing gross residual tumor when performed during days 1 to 3 after the resection of a preoperatively enhancing high-grade glioma. This timing avoided surgically induced contrast enhancement and minimized interpretative difficulties [1]. FLAIR is often the diagnostic modality in the early postoperative period due to its better delineation of enhancing residual tissue at the border of the resection cavity [7]. According to Sinan, et al. early postoperative FLAIR imaging is less reliable to determine the extent of non-enhancing residual glioma as it overestimates the residual tumor volume due to resection induced ischemia and hence, restricted diffusion volumes are imperative $[8,9]$. T2 FLAIR imaging has limitations in that it cannot differentiate infiltrating tumor from vasogenic edema reliably as both are hyperintense on T2 FLAIR sequences.

According to Smith JS, et al. a new enhancement observed after glioma surgery could be confused with recurrent tumor and interpreted as early treatment failure. Therefore, any new area of contrast enhancement post-operatively should be interpreted in the context of the immediate postoperative DWI, a sequence that is essential and should be a part of any routine brain tumor imaging protocol [10]. According to Kessler and coworkers, the most common etiologies of diffusion restriction include abnormally high cellularity, cellular injury, and peritumoral edema. Any new area of low ADC signal on post-operative imaging should raise the suspicion for tumor recurrence/progression [3] (Figure 2).

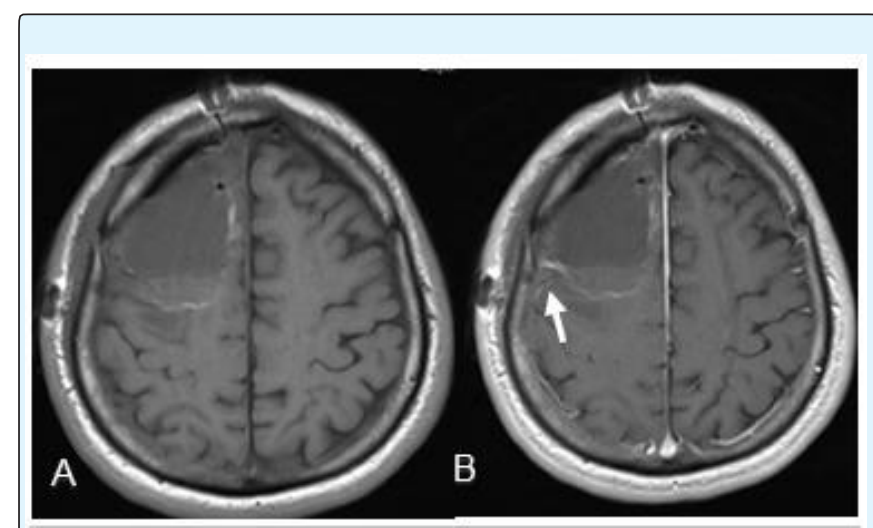

Figure 2: EPMRI (24 hours post op) A: Axial T1 weighted B: Axial T1-post contrast Status post frontal craniotomy for grade IV glioblastoma resection Edema and hemorrhage along resection cavity representing post-surgical changes.

Post-operative cellular injury is frequently due to direct surgical trauma, vascular injury, and devascularization of tumor [11]. One study demonstrated new reduced diffusion in or around the resection cavity in $64 \%$ of resected gliomas, of which $93 \%$ went on to develop encephalomalacia on serial imaging, suggesting cellular injury [10]. It is therefore very important to correlate any new enhancement with the immediate postoperative DWI so as not to erroneously diagnose tumor progression when it is in fact, postoperative injury. Post-operative cytotoxic edema frequently shows up as contrast enhancement in the subacute phase that resolves on follow-up imaging as encephalomalacia forms [3].

Some researchers reported that ADC maps are not useful for reliably demarcating brain tumor boundaries due to the presence of vasogenic edema $[9,12,13]$. In these cases, DWI must be combined with other advanced imaging techniques. According to Kessler AT, et al. PWI is particularly important on post-treatment imaging, as 


\section{Clinical Radiology \& Imaging Journal}

areas of increased perfusion can be suggestive of tumor growth or recurrence. On DSC perfusion, any new area of elevated $\mathrm{rCBV}$ under signal intensity time curve may be a marker for tumor growth/ recurrence [3]. A pitfall of DSC perfusion is that $\mathrm{rCBV}$ is calculated with the underlying assumption that there is no contrast leakage or recirculation. However, there is always some degree of contrast leakage due to brain tumor violation of the blood-brain barrier.

\section{References}

1. Albert FK, Forsting M, Sartor K, Adams HP, Kunze S (1994) Early postoperative magnetic resonance imaging after resection of malignant glioma: objective evaluation of residual tumor and its influence on regrowth and prognosis. Neurosurgery 34(1): 45-60.

2. Becker G, Hofmann E, Woydt M, Hulsmann U, Maurer M, et al. (1999) Postoperative neuroimaging of highgrade gliomas: comparison of transcranial sonography, magnetic resonance imaging, and computed tomography. Neurosurgery 44(3): 469477.

3. Kessler AT, Bhatt AA (2018) Brain tumour posttreatment imaging and treatment-related complications. Insights Imaging. 9(6): 1057-1075.

4. Elster AD, DiPersio DA (1990) Cranaial postoperative site: assessment with contrast-enhanced MR imaging. Radiology 174(1): 93-98.

5. Forsting M, Albert FK, Kunze S, Adams HP, Zenner D, et al. (1993) Extirpation of glioblastomas: MR and CT follow-up of residual tumor and regrowth patterns. Am J Neuroradiol 14(1): 77-87.

6. Knauth M, Aras N, Wirtz CR, Dörfler A, Engelhorn T, et al. (1999) Surgically induced intracranial contrast enhancement: potential source of diagnostic error in intraoperative MR imaging. Am J Neuroradiol 20(8): 1547-1553.

7. Essig M, Metzner R, Bonsanto M, Hawighorst $\mathrm{H}$, Debus J, et al. (2001) Postoperative fluid-attenuated inversion recovery MR imaging of cerebral gliomas: initial results. Eur Radiol 11(10): 2004-2010.

8. Belhawi SM, Hoefnagels FW, Baaijen JC, Aliaga ES, Reijneveld JC, et al. (2011) Early postoperative MRI overestimates residual tumour after resection of gliomas with no or minimal enhancement. Eur Radiol 21(7): 1526-1534.

9. Pauleit D, Langen KJ, Floeth F, Hautzel H, Riemenschneider MJ, et al. (2004) Can the apparent diffusion coefficient be used as a noninvasive parameter to distinguish tumor tissue from peritumoral tissue in cerebral gliomas? J Magn Reson Imaging 20(5): 758-764.

10. Smith JS, Chang EF, Lamborn KR, Chang SM, Prados MD, et al. (2008) Role of extent of resection in the long-term outcome of low-grade hemispheric gliomas. J Clin Oncol 26(8): 1338-1345.

11. Cha $S$ (2006) Update on brain tumor imaging: from anatomy to physiology. AJNR Am J Neuroradiol 27(3): 475-487.

12. Castillo M, Smith JK, Kwock L, Wilber K (2001) Apparent diffusion coefficients in the evaluation of high-grade cerebral gliomas. AJNR Am J Neuroradiol 22(1): 60-64.

13. Stadnik TW, Chaskis C, Michotte A, Shabana WM, van Rompaey K, et al. (2001) Diffusion-weighted MR imaging of intracerebral masses: comparison with conventional MR imaging and histologic findings. AJNR Am J Neuroradiol 22(5): 969-976. 